\title{
On Price Equilibrium with Multi-Product Firms
}

\author{
Zsolt Sándor ${ }^{1}$ \\ Erasmus University Rotterdam
}

\begin{abstract}
In this paper we provide a result that shows existence and uniqueness of Nash equilibrium in cases in which existent methods are problematic to apply. We employ this result to the model with simple logit demand, and show existence and uniqueness of price equilibrium when firms produce multiple non-symmetric products. Our proof for this case is based only on the intuitive assumption that market shares are decreasing in own price.
\end{abstract}

JEL No.: D43

Key words: Nash-Bertrand, price competition, product differentiation, logit.

Econometric Institute Report EI 2004-50

\footnotetext{
${ }^{1}$ Econometric Institute, Erasmus University Rotterdam, P.O. Box 1738, 3000 DR Rotterdam, The Netherlands. E-mail: sandor@few.eur.nl, phone: +31 10408 1416, fax: +31 104089162.

The author thanks Mark Armstrong and Frank Verboven for helpful remarks.
} 


\section{Introduction}

Price equilibrium models with differentiated products have received much attention recently especially in the empirical industrial organization literature. Important examples of this literature are the works by Berry, Levinsohn and Pakes (1995), Feenstra and Levinsohn (1995) and Nevo (2001). The models in these studies use pricing assumptions in order to estimate the model parameters. Existence of price equilibrium is a necessary condition for the identification of the parameters. Uniqueness of price equilibrium is important from a practical point of view since it is a requirement for applying the structural empirical approach for policy analysis via simulation estimators. Uniqueness of equilibrium is also useful for efficient estimation because it is a necessary condition for constructing the efficient instruments (Sándor, 2001, Berry, Linton and Pakes, 2003).

Existence and uniqueness of price equilibrium is also important from a theoretical point of view. Caplin and Nalebuff (1991) establish price equilibrium existence results for rather general model specifications and uniqueness results for some particular cases. Anderson, de Palma and Thisse (1992) provide a review of equilibrium results for models with logit demand. Peitz (2000) extends the results of Caplin and Nalebuff (1991) to cases that can be viewed as more realistic, like utility maximization with a budget constraint or boundedly rational consumers. Mizuno (2003) extends the uniqueness results of Caplin and Nalebuff (1991). All these studies assume that firms produce one product and their approaches cannot be easily generalized to multi-product firms. Anderson and de Palma (1992) consider multi-product firms and show the existence and uniqueness of price equilibrium in a model with nested logit demand and symmetric products. This latter feature makes this result so specific that it cannot be generalized to a model with realistic non-symmetric products. Another way to deal with multi-product firms is shown by Milgrom and Roberts (1990), who study supermodular and log-supermodular games. However, the pricing games involved in empirically relevant models do not necessarily satisfy these properties. 
In this paper we provide a result that can serve as a tool for showing existence and uniqueness of price equilibrium in some cases in which the approaches mentioned above cannot. This is the topic of the next section. We apply this result to a simple version of the models from Berry, Levinsohn and Pakes (1995) and Nevo (2001), the model with simple logit demand, and show existence and uniqueness of price equilibrium when firms produce multiple products without the symmetry property. The simple logit demand model, although empirically less relevant since it generates restrictive substitution patterns, is often used to illustrate various estimation features (e.g., Berry, Levinsohn and Pakes, 1995) and in Monte Carlo simulations (e.g., Berry, Linton and Pakes, 2003). In spite of the fact that it is known as a well-behaved model, no proof of existence and uniqueness of price equilibrium has been established in the literature. For example, Berry, Linton and Pakes (2003, p.15) mention that price equilibrium for the simple logit is known to be unique only when each product is owned by a different firm. We present the proof of existence and uniqueness in section 3, and we make some final remarks in section 4.

\section{An equilibrium result}

In this section we briefly discuss the available theoretical results regarding existence and uniqueness of price equilibrium, and then present our result. The classical approach for showing existence is based on the result according to which games with convex and compact strategy sets and quasi-concave pay-off functions have a pure strategy Nash equilibrium. The Nash equilibrium is obtained as the fixed point of the best reply by applying the Brouwer or Kakutani fixed point theorem. This approach is taken by Caplin and Nalebuff (1991), who use results on generalized concavity of probabilities to prove that the profit functions are quasi-concave. For multi-product firms quasi-concavity of the profit functions is difficult to verify with this method, while a direct proof is typically hard to obtain.

An essentially different approach is supermodularity. Supermodularity of games 
was applied by Milgrom and Roberts (1990) to a number of equilibrium problems. These authors establish among others that with one-product firms the pricing game corresponding to the model with simple logit demand is log-supermodular and therefore has a unique price equilibrium. Supermodularity or log-supermodularity, as they show, guarantees the existence of equilibrium also in games where the strategy sets are multi-dimensional, but it appears that in general the pricing games arising from the empirical models of interest do not satisfy these properties. An example underpinning this statement is the model with simple logit demand, as we show in Appendix B.

Uniqueness of the price equilibrium is typically established by proving that the second derivative of the profit function has the dominant diagonal property. This condition, however, appears to be too strong for the models that we consider, and therefore we use a more general approach. For a detailed exposition of methods for proving Nash equilibrium existence and uniqueness we refer to Vives (1999).

Our approach is different from those discussed above in that we consider the price equilibrium as the solution of the first order conditions of profit maximization and not as the fixed point of the best reply function (or correspondence). Hence we establish that the first order conditions have a solution and show that any such solution is a Nash equilibrium of the game. For this we show that a player's strategy corresponding to a solution is the best reply to the other players' strategies corresponding to this solution. Uniqueness follows from the uniqueness of the solution to the first order conditions.

We turn now to the formal exposition. Assume a game with a finite number of players denoted $f=1, \ldots, F$ whose strategies are multi-dimensional real convex compact sets $D_{f}$. Let $\Pi_{f}: D \rightarrow \mathbb{R}$ denote their continuously differentiable profit functions, where $D=D_{1} \times \ldots \times D_{F}$. We use the common notation that $\mathbf{v}_{-j}$ is the vector $\mathbf{v}$ without its $j$ 'th component, $\mathbf{v}_{-f}$ is the part of vector $\mathbf{v}$ without the components corresponding to the vector $\mathbf{v}_{f}$. By the notation of multi-dimensional intervals that are open on one side we mean $[\mathbf{a}, \mathbf{b}) \equiv\left[a_{1}, b_{1}\right) \times \ldots \times\left[a_{n}, b_{n}\right)$ where 
$\mathbf{a}=\left(a_{1}, \ldots, a_{n}\right)^{\prime}$ and $\mathbf{b}=\left(b_{1}, \ldots, b_{n}\right)^{\prime}$. Vectors are by default column vectors, and, in order to avoid confusion, they are denoted by boldface letters.

The result on which our equilibrium existence and uniqueness is based is the following.

Proposition 1 Consider a game for which there is a strategy $\mathbf{p}^{*}$ that satisfies the conditions:

1. For any $f \in\{1, \ldots, F\} \frac{\partial \Pi_{f}\left(\mathbf{p}^{*}\right)}{\partial \mathbf{p}_{f}}=0$.

2. For any $f \in\{1, \ldots, F\}$ there is exactly one $\mathbf{p}_{f}$ for which $\frac{\partial \Pi_{f}\left(\mathbf{p}_{f}, \mathbf{p}_{-f}^{*}\right)}{\partial \mathbf{p}_{f}}=0$.

3. For any $f \in\{1, \ldots, F\}, \Pi_{f}\left(\mathbf{p}_{f}, \mathbf{p}_{-f}^{*}\right)$ has an interior global maximum with respect to $\mathbf{p}_{f} \in D_{f}$.

Then $\mathbf{p}^{*}$ is a Nash equilibrium of the game. If, in addition, there is a unique $\mathbf{p}^{*}$ satisfying condition 1, then it is the unique Nash equilibrium of the game.

Proof. In order for $\mathbf{p}^{*}$ to be a Nash equilibrium it should satisfy that $\Pi_{f}\left(\mathbf{p}_{f}^{*}, \mathbf{p}_{-f}^{*}\right) \geq$ $\Pi_{f}\left(\mathbf{p}_{f}, \mathbf{p}_{-f}^{*}\right)$ for any $\mathbf{p}_{f} \in D_{f}$. By condition 3 there is a $\overline{\mathbf{p}}_{f} \in D_{f}$ that is an interior global maximum point of $\Pi_{f}\left(\cdot, \mathbf{p}_{-f}^{*}\right)$. This satisfies $\frac{\partial \Pi_{f}\left(\overline{\mathbf{p}}_{f}, \mathbf{p}_{-f}^{*}\right)}{\partial \mathbf{p}_{f}}=0$. By condition 1 we know that $\frac{\partial \Pi_{f}\left(\mathbf{p}_{f}^{*}, \mathbf{p}_{-f}^{*}\right)}{\partial \mathbf{p}_{f}}=0$ also holds. Then by $2 \overline{\mathbf{p}}_{f}=\mathbf{p}_{f}^{*}$ and hence $\mathbf{p}^{*}$ satisfies $\Pi_{f}\left(\mathbf{p}_{f}^{*}, \mathbf{p}_{-f}^{*}\right) \geq \Pi_{f}\left(\mathbf{p}_{f}, \mathbf{p}_{-f}^{*}\right)$, so it is a Nash equilibrium. Since any Nash equilibrium of the game necessarily satisfies condition 1, the uniqueness follows.

We note that conditions 2 and 3 of the proposition can be viewed as a generalization of strict quasi-concavity of the profit function and they imply that there is a unique best reply to the equilibrium strategies.

We introduce a function $\mathbf{g}$ whose fixed points are exactly the solutions of the first order conditions of profit maximization, that is, $\mathbf{g}(\mathbf{p})=\mathbf{p}$ if and only if $\frac{\partial \Pi_{f}(\mathbf{p})}{\partial \mathbf{p}_{f}}=0$ for any $f \in\{1, \ldots, F\}$. In order to demonstrate that conditions 1 and 2 of Lemma 
1 are satisfied we use a fixed point uniqueness result, which is an implication of Kellogg's (1976) result.

Lemma 2 (Kellogg, 1976) Let $\mathbf{g}: D \rightarrow D$ be a continuously differentiable function on a convex compact set $D \subset \mathbb{R}^{n}$. If $\operatorname{det}\left(\frac{\partial \mathbf{g}(\mathbf{p})}{\partial \mathbf{p}^{\prime}}-I_{n}\right) \neq 0$ for any $\mathbf{p} \in D$, and $\mathbf{g}$ has no fixed points on the boundary of $D$ then $\mathbf{g}$ has a unique fixed point.

This result is used twice in the proof: once for existence and once for uniqueness. Existence is based on the uniqueness of the profit maximizing solution. In order to show that condition 3 holds we use the next result, proved in Appendix A.

Lemma 3 Assume that the profit function $\Pi_{f}$ of firm $f$ is defined on the interval $[\mathbf{c}, \mathbf{H}]$, and there exists a vector $\overline{\mathbf{p}}_{f} \in\left(\mathbf{c}_{f}, \mathbf{H}_{f}\right)$ such that for any $j \in G_{f}$, any $p_{j} \in\left[\bar{p}_{j}, H_{j}\right]$ and any $\mathbf{p}_{-j} \in\left[\mathbf{c}_{-j}, \mathbf{H}_{-j}\right]$ we have

$$
\frac{\partial \Pi_{f}}{\partial p_{j}}\left(p_{j}, \mathbf{p}_{-j}\right)<0 \quad \text { and } \quad \frac{\partial \Pi_{f}}{\partial p_{j}}\left(c_{j}, \mathbf{p}_{-j}\right)>0 \text {. }
$$

Then for any $\mathbf{p}_{f} \notin\left(\mathbf{c}_{f}, \overline{\mathbf{p}}_{f}\right]$ and for any $\mathbf{p}_{-f} \in\left[\mathbf{c}_{-f}, \mathbf{H}_{-f}\right]$ there is a $\widetilde{\mathbf{p}}_{f} \in\left(\mathbf{c}_{f}, \overline{\mathbf{p}}_{f}\right]$ such that

$$
\Pi_{f}\left(\widetilde{\mathbf{p}}_{f}, \mathbf{p}_{-f}\right)>\Pi_{f}\left(\mathbf{p}_{f}, \mathbf{p}_{-f}\right)
$$

An implication of this lemma is that a profit function which satisfies these conditions has interior global maximum. The lemma, in words, states that if such a profit function is decreasing in the prices of the firm beyond a certain bound and increasing at the marginal cost values, then the global maximum of the profit function is attained at points that are kept between some bounds. These bounds prevent the profit function from having a global maximum on the boundary of its definition domain.

\section{The simple logit case}

Suppose that there are $J$ products in the market denoted $1, \ldots, J$. For $j \in\{1, \ldots, J\}$, let $d_{j}$ and $p_{j}$ denote the characteristics and the price of product $j$, respectively. The 
utility of an individual $i$ who purchases product $j$ is

$$
\begin{aligned}
& u_{i j}=-\alpha p_{j}+d_{j}+\varepsilon_{i j}, \\
& u_{i 0}=\varepsilon_{i 0},
\end{aligned}
$$

where $\alpha$ is a scalar parameter, $\varepsilon_{i j}$ is an iid type I extreme value random variable, and product 0 represents the alternative when no product is purchased. In empirical studies $d_{j}$ is typically taken as a linear function of several characteristics of product $j$. The probability that product $j$ is purchased, which we regard as the market share of product $j$, is

$$
s_{j}=\frac{\exp \left(-\alpha p_{j}+d_{j}\right)}{1+\sum_{r=1}^{J} \exp \left(-\alpha p_{r}+d_{r}\right)} .
$$

Due to the simplicity of this formula the simple logit model is often used for illustrating various issues regarding discrete choice. From an empirical point of view it is well known that its applicability is limited due to the restrictive substitution patterns that it generates.

Suppose further that the $J$ products are produced by $F$ firms and each firm $f \in\{1, \ldots, F\}$ sells a subset $G_{f}$ of the $J$ products. The profit of firm $f$ is

$$
\Pi_{f}=\sum_{j \in G_{f}}\left(p_{j}-c_{j}\right) s_{j}
$$

where $c_{j}$ denotes the constant marginal cost of producing product $j$. The first order conditions for profit maximization are equivalent to the system of equations in $\mathbf{p}=\left(p_{1}, \ldots, p_{J}\right)^{\prime}$

$$
\mathbf{p}_{f}-\mathbf{c}_{f}=\frac{1}{\alpha} \frac{\iota_{f}}{1-\mathbf{s}_{f}^{\prime} \iota_{f}} \quad \text { for } f=1, \ldots, F,
$$

where $\mathbf{p}_{f}, \mathbf{c}_{f}$ and $\mathbf{s}_{f}$ are the price, marginal cost and market share vectors corresponding to the products of firm $f$, and $\iota_{f}$ is the vector of ones with $\left|G_{f}\right|$ number of elements. This system of equations implies that the function $\mathbf{g}$ whose fixed points are the solutions of the first order conditions of profit maximization should 
be defined as

$$
\begin{aligned}
\mathbf{g}_{f}(\mathbf{p}) & =\mathbf{c}_{f}+\frac{1}{\alpha} \frac{\iota_{f}}{1-\mathbf{s}_{f}^{\prime} \iota_{f}} \text { for } f=1, \ldots, F, \text { and } \\
\mathbf{g}(\mathbf{p}) & =\left(\mathbf{g}_{1}(\mathbf{p})^{\prime}, \ldots, \mathbf{g}_{F}(\mathbf{p})^{\prime}\right)^{\prime}
\end{aligned}
$$

Below we show that this pricing game has a unique Nash equilibrium. The only necessary assumption is the intuitive $\alpha>0$. The proof of the final result relies on Proposition 1.

First we show the existence of a $J$-dimensional compact interval that $\mathbf{g}$ transforms into itself. Then we verify that $\mathbf{g}$ satisfies the conditions of Lemma 2. This way we show that conditions 1 and 2 of Proposition 1 hold. Finally we show that the conditions of Lemma 3 are satisfied, which on its turn implies condition 3 of Proposition 1.

We start by demonstrating the conditions of Lemma 2. The function $\mathbf{g}$ from (4) has the components

$$
g_{j}(\mathbf{p})=c_{j}+\frac{1}{\alpha} \frac{1}{1-\mathbf{s}_{f}^{\prime} \iota_{f}} \text { for any } f \text { and } j \in G_{f}
$$

We define

$$
B_{j} \equiv c_{j}+\frac{1}{\alpha} \frac{1}{s_{0}(\mathbf{c})} \quad \text { for any } j \in\{1, \ldots, J\},
$$

where $s_{0}(\mathbf{c})$ is the probability of the no-purchase alternative computed for $\mathbf{p}=\mathbf{c}$. The following result (proved in Appendix A) shows that there is a compact interval which is transformed by $\mathbf{g}$ into itself, and there is no fixed point of $\mathbf{g}$ on the boundary of this interval.

Proposition 4 For any $\mathbf{p} \in\left[c_{1}, \infty\right) \times \ldots \times\left[c_{J}, \infty\right) g_{j}(\mathbf{p})$ satisfies

$$
c_{j}<g_{j}(\mathbf{p})<B_{j} .
$$

Next we establish the nonsingularity of the Jacobian of $\mathbf{g}$ minus the identity matrix. This then completes the proof of the conditions of Lemma 2.

Proposition $\mathbf{5}$ The function $\mathbf{g}$ is continuously differentiable on $\mathbb{R}^{J}$ and for any $\mathbf{p} \in \mathbb{R}^{J}$ 

1. $\operatorname{det}\left(\frac{\partial \mathbf{g}_{f}(\mathbf{p})}{\partial \mathbf{p}_{f}^{\prime}}-I_{f}\right) \neq 0$ for any firm $f$
2. $\operatorname{det}\left(\frac{\partial \mathbf{g}(\mathbf{p})}{\partial \mathbf{p}^{\prime}}-I_{J}\right) \neq 0$. (Proved in Appendix A.)

Finally, we prove that the conditions of Lemma 3 are satisfied.

Proposition 6 For any firm $f$ and any $j \in G_{f}$ there exists a $\bar{p}_{j}>c_{j}$ such that

$$
\frac{\partial \Pi_{f}}{\partial p_{j}}\left(p_{j}, \mathbf{p}_{-j}\right)<0 \text { and } \frac{\partial \Pi_{f}}{\partial p_{j}}\left(c_{j}, \mathbf{p}_{-j}\right)>0
$$

for any $p_{j} \geq \bar{p}_{j}$ and $\mathbf{p}_{-j} \geq \mathbf{c}_{-j}$.

Proof. The derivative of $\Pi_{f}$ with respect to $p_{j}$ can be written as

$$
\frac{\partial \Pi_{f}(\mathbf{p})}{\partial p_{j}}=s_{j}\left(1-\alpha\left(p_{j}-c_{j}\right)+\alpha \Pi_{f}(\mathbf{p})\right) .
$$

First we note that the profit $\Pi_{f}(\mathbf{p})$ is bounded in $\mathbf{p}$. This can be seen from

$$
\Pi_{f}(\mathbf{p})=\sum_{j \in G_{f}}\left(p_{j}-c_{j}\right) \frac{\exp \left(-\alpha p_{j}+d_{j}\right)}{1+\sum_{r=1}^{J} \exp \left(-\alpha p_{r}+d_{r}\right)} \leq \sum_{j \in G_{f}}\left(p_{j}-c_{j}\right) \exp \left(-\alpha p_{j}+d_{j}\right)
$$

for $p_{j} \geq c_{j}$ and the fact that $\exp \left(-\alpha p_{j}+x_{j}^{\prime} \beta+\xi_{j}\right)\left(p_{j}-c_{j}\right) \underset{p_{j} \rightarrow \infty}{\rightarrow} 0$ because $\alpha>0$. Then it follows that for large $p_{j}$ the right hand side of the inequality below is negative:

$$
\frac{\partial \Pi_{f}(\mathbf{p})}{\partial p_{j}}<1-\alpha\left(p_{j}-c_{j}\right)+\alpha \sup \Pi_{f}(\mathbf{p}) .
$$

This implies the existence of $\bar{p}_{j}$ with the announced property.

The second inequality from the statement of the proposition follows directly from (5).

The final result regarding the existence and uniqueness of price equilibrium is contained in the following statement.

Theorem 7 In the simple logit model if $\alpha>0$ there exists a unique price equilibrium in $\left[c_{1}, \infty\right) \times \ldots \times\left[c_{J}, \infty\right)$. 
Proof. The proof is based on Proposition 1. For each $j \in\{1, \ldots, J\}$ we define $K_{j}$ such that

$$
K_{j} \geq \max \left\{\bar{p}_{j}, B_{j}\right\}
$$

Let the profit function of firm $f$ be defined on $\left[\mathbf{c}_{f}, \mathbf{K}_{f}\right]$. Then Lemma 3 implies that $\Pi_{f}\left(\cdot, \mathbf{p}_{-f}\right):\left[\mathbf{c}_{f}, \mathbf{K}_{f}\right] \rightarrow \mathbb{R}$ has interior global maximum. So the profit functions are defined on a convex compact set, are continuously differentiable and satisfy condition 3 of Lemma 1.

Proposition 4 implies that $\mathbf{g}(\mathbf{p}) \in(\mathbf{c}, \mathbf{B}) \subset[\mathbf{c}, \mathbf{K}]$ for any $\mathbf{p} \in[\mathbf{c}, \mathbf{K}]$, and therefore $\mathbf{g}$ does not have any fixed point on the boundary of $[\mathbf{c}, \mathbf{K}]$. Together with part (2) of Proposition 5 this implies that the conditions of Kellogg's fixed point theorem (Lemma 2) are satisfied and therefore $\mathbf{g}$ has a unique fixed point. This establishes condition 1 of Proposition 1 and the uniqueness of $\mathbf{p}^{*}$ satisfying this condition. Condition 2 of this proposition follows by repeating the previous arguments for $\mathbf{g}_{f}\left(\cdot, \mathbf{p}_{-f}^{*}\right)$ applying now part (1) of Proposition 5.

So we have obtained that the conditions of Proposition 1 are satisfied. Hence there is a unique price equilibrium in the set $[\mathbf{c}, \mathbf{K}]$. Since this statement is true for any $\mathbf{K}$ with the property (6), it follows that there is exactly one price equilibrium in the set $\left[c_{1}, \infty\right) \times \ldots \times\left[c_{J}, \infty\right)$.

\section{Final remarks}

We have presented a result that shows existence and uniqueness of Nash equilibrium in some situations in which previously used results cannot. We applied this result to the model with simple logit demand. Adopting realistic assumptions like multiproduct firms and non-symmetric products, we have shown that the pricing game in this model has a unique Nash equilibrium. The only condition used in the proof is that market shares of products are decreasing in own price.

In the proofs of both existence and uniqueness of price equilibrium we employed Kellogg's (1976) fixed point theorem. There is a connection between this approach and the so-called global univalence approach of Gale and Nikaido (1965). It turns 
out that application of the former comes down to verifying conditions similar to the case when we apply the latter. We refer to Vives (1999, p.47-48) for more details on the latter approach.

The simple logit model is among the simplest models of discrete choice demand. Yet we could not apply the methods established in the literature for proving the existence of price equilibrium in the multi-product case. This is because, on the one hand, the pricing game implied is not (log-)supermodular, and on the other hand, due to multi-dimensionality of the profit functions, their quasi-concavity appears difficult to judge. This remains to be an interesting puzzle.

Continuing the remarks on the complexity of our proof, we mention that a powerful tool used extensively to prove uniqueness in general equilibrium problems, is the so-called index theory (see, e.g., Mas-Colell, 1985 for a fairly detailed presentation). This theory can also be applied in the framework of the present paper, and it offers a more general approach. But in spite of the generalization offered, this approach does not significantly simplify the proof. Neither does the additional observation that the Hessians of the profit functions evaluated at the solutions to the first order conditions are negative definite (because they are diagonal matrices with negative diagonal elements; see equation (11) in Appendix B). From index theory it follows that the first order conditions have a unique interior solution, but it is not possible to avoid the proof that the profits have interior global maximum points.

\section{Appendix A}

Proof of Lemma 3. Take an arbitrary $\mathbf{p}_{f} \notin\left(\mathbf{c}_{f}, \overline{\mathbf{p}}_{f}\right]$. For any $j \in G_{f}$ define $\widetilde{\mathbf{p}}_{f}$ by its components

$$
\widetilde{p}_{j}= \begin{cases}\bar{p}_{j}, & \text { if } p_{j}>\bar{p}_{j} \\ c_{j}+\varepsilon_{j}, & \text { if } p_{j}=c_{j} \\ p_{j}, & \text { otherwise }\end{cases}
$$


where $\varepsilon_{j}>0$ will be specified below. Denote the products of firm $f$ by $f 1, f 2, \ldots, f L$. Then we can show that

$$
\begin{aligned}
\Pi_{f}\left(\widetilde{p}_{f 1}, \ldots, \widetilde{p}_{f L}, \mathbf{p}_{-f}\right) & \geq \Pi_{f}\left(p_{f 1}, \widetilde{p}_{f 2}, \ldots, \widetilde{p}_{f L}, \mathbf{p}_{-f}\right) \\
& \geq \Pi_{f}\left(p_{f 1}, p_{f 2}, \widetilde{p}_{f 3}, \ldots, \widetilde{p}_{f L}, \mathbf{p}_{-f}\right) \\
& \geq \ldots \geq \Pi_{f}\left(p_{f 1}, \ldots, p_{f L}, \mathbf{p}_{-f}\right)
\end{aligned}
$$

step by step using (1). For example, we can show that the first inequality holds by treating the different cases for $p_{f 1}$ separately. If $p_{f 1} \in\left(c_{f 1}, \bar{p}_{f 1}\right]$ then $\widetilde{p}_{f 1}=p_{f 1}$, so there is nothing to prove. If $p_{f 1}>\bar{p}_{f 1}$ then by the first inequality from (1) we have that $\Pi_{f}\left(\cdot, \widetilde{p}_{-f 1}, \mathbf{p}_{-f}\right)$ is strictly decreasing and hence $\Pi_{f}\left(\widetilde{p}_{f 1}, \widetilde{p}_{-f 1}, \mathbf{p}_{-f}\right)=$ $\Pi_{f}\left(\bar{p}_{f 1}, \widetilde{p}_{-f 1}, \mathbf{p}_{-f}\right)>\Pi_{f}\left(p_{f 1}, \widetilde{p}_{-f 1}, \mathbf{p}_{-f}\right)$. If $p_{f 1}=c_{f 1}$ then by the second inequality from $(1) \Pi_{f}\left(\cdot, \widetilde{p}_{-f 1}, \mathbf{p}_{-f}\right)$ is strictly increasing and therefore there is a small $\varepsilon_{f 1}>0$ for which $\widetilde{p}_{f 1}=c_{f 1}+\varepsilon_{f 1}$ satisfies

$$
\Pi_{f}\left(\widetilde{p}_{f 1}, \widetilde{p}_{-f 1}, \mathbf{p}_{-f}\right)>\Pi_{f}\left(c_{f 1}, \widetilde{p}_{-f 1}, \mathbf{p}_{-f}\right)=\Pi_{f}\left(p_{f 1}, \widetilde{p}_{-f 1}, \mathbf{p}_{-f}\right)
$$

For showing the other steps of inequality (7) we proceed similarly. The strict inequality from (2) is implied by the fact that if $\mathbf{p}_{f} \notin\left(\mathbf{c}_{f}, \overline{\mathbf{p}}_{f}\right]$ then at least one component of $\mathbf{p}_{f}$, say $j$, satisfies that $p_{j} \notin\left(c_{j}, \bar{p}_{j}\right]$.

Lemma 8 Let $M$ be an $F \times F$ non-singular matrix, $\lambda$ a scalar and $u$ and $v$ column vectors of size $F$. Then

$$
\operatorname{det}\left(M-\lambda u v^{\prime}\right)=\left(1-\lambda v^{\prime} M^{-1} u\right) \operatorname{det} M
$$

and hence the matrix $M-\lambda u v^{\prime}$ is non-singular if and only if $1-\lambda v^{\prime} M^{-1} u \neq 0$. If this last non-equality holds, then

$$
\left(M-\lambda u v^{\prime}\right)^{-1}=M^{-1}+\frac{\lambda}{1-\lambda v^{\prime} M^{-1} u} M^{-1} u v^{\prime} M^{-1} .
$$

(For a proof we refer to Dhrymes, 1984, p.40.)

Proof of Proposition 4. The first part of the inequality is obvious. For the second part, because the probabilities of all alternatives sum to one, and $s_{0}$ is 
increasing in $\mathbf{p}$, we have

$$
g_{j}(\mathbf{p})=c_{j}+\frac{1}{\alpha} \frac{1}{s_{0}(\mathbf{p})+\sum_{r \notin G_{f}} s_{r}(\mathbf{p})}<c_{j}+\frac{1}{\alpha} \frac{1}{s_{0}(\mathbf{p})} \leq c_{j}+\frac{1}{\alpha} \frac{1}{s_{0}(\mathbf{c})} .
$$

Proof of Proposition 5. The partial derivatives of the components of $\mathbf{g}$ corresponding to a firm $f$ have the expressions

$$
\begin{aligned}
\frac{\partial g_{j}}{\partial p_{h}} & =-\frac{s_{h}}{1-\mathbf{s}_{f}^{\prime} \iota_{f}} \quad \text { for } j, h \in G_{f}, \quad \text { and } \\
\frac{\partial g_{j}}{\partial p_{k}} & =\frac{s_{k}}{1-\mathbf{s}_{f}^{\prime} \iota_{f}} \frac{\mathbf{s}_{f}^{\prime} \iota_{f}}{1-\mathbf{s}_{f}^{\prime} \iota_{f}} \quad \text { for } j \in G_{f}, k \notin G_{f} .
\end{aligned}
$$

These imply that the diagonal blocks of the derivatives matrix are

$$
\frac{\partial \mathbf{g}_{f}}{\partial \mathbf{p}_{f}^{\prime}}=-\frac{1}{1-\mathbf{s}_{f}^{\prime} \iota_{f}} \iota_{f} \mathbf{s}_{f}^{\prime}, \quad f=1, \ldots, F .
$$

Lemma 8 (from Appendix A) implies that the matrix $\frac{\partial \mathbf{g}_{f}}{\partial \mathbf{p}_{f}^{\prime}}-I_{f}=-\left(I_{f}+\frac{1}{1-\mathbf{s}_{f}^{\prime} \iota_{f}} \iota_{f} \mathbf{s}_{f}^{\prime}\right)$ is non-singular if $1+\frac{\mathbf{s}_{f}^{\prime} \iota_{f}}{1-\mathbf{s}_{f}^{\prime} \iota_{f}} \neq 0$, which is satisfied. This proves the first statement of the proposition.

For the second statement we compute the off-diagonal blocks of the derivatives matrix, which are

$$
\frac{\partial \mathbf{g}_{f}}{\partial \mathbf{p}_{q}^{\prime}}=\frac{\mathbf{s}_{f}^{\prime} \iota_{f}}{\left(1-\mathbf{s}_{f}^{\prime} \iota_{f}\right)^{2}} \iota_{q} \mathbf{s}_{q}^{\prime}, \quad f, q=1, \ldots, F, f \neq q .
$$

To simplify the involved expressions we introduce the notation:

$$
\rho_{f} \equiv \frac{\mathbf{s}_{f}^{\prime} \iota_{f}}{\left(1-\mathbf{s}_{f}^{\prime} \iota_{f}\right)^{2}} \quad \text { and } \quad \psi_{f} \equiv \frac{1}{\left(1-\mathbf{s}_{f}^{\prime} \iota_{f}\right)^{2}}, \quad \text { for } f=1, \ldots, F .
$$

We can write $\frac{\partial \mathbf{g}}{\partial \mathbf{p}^{\prime}}-I_{J}$ in the form

$$
\frac{\partial \mathbf{g}}{\partial \mathbf{p}^{\prime}}-I_{J}=\left[\begin{array}{c}
\rho_{1} \iota_{1} \\
\vdots \\
\rho_{F} \iota_{F}
\end{array}\right]\left[\begin{array}{c}
\mathbf{s}_{1} \\
\vdots \\
\mathbf{s}_{F}
\end{array}\right]^{\prime}-\left[\begin{array}{ccc}
\psi_{1} \iota_{1} \mathbf{s}_{1}^{\prime}+I_{1} & \cdots & 0 \\
\vdots & \ddots & \vdots \\
0 & \cdots & \psi_{F} \iota_{F} \mathbf{s}_{F}^{\prime}+I_{F}
\end{array}\right],
$$

where the matrix on the right hand side is block-diagonal. A diagonal block of this matrix $\psi_{f} \iota_{f} \mathbf{s}_{f}^{\prime}+I_{f}$ is invertible if $1+\psi_{f} \mathbf{s}_{f}^{\prime} \iota_{f} \neq 0$ for any $\mathbf{p} \in \mathbb{R}^{J}$ (Lemma 8). This property is clearly satisfied. Then its inverse is

$$
\left(\psi_{f} \iota_{f} \mathbf{s}_{f}^{\prime}+I_{f}\right)^{-1}=I_{f}-\frac{\psi_{f}}{1+\psi_{f} \mathbf{s}_{f}^{\prime} \iota_{f}} \iota_{f} \mathbf{s}_{f}^{\prime} .
$$


From (8) and Lemma 8 the matrix $\frac{\partial \mathbf{g}}{\partial \mathbf{p}^{\prime}}-I_{J}$ is non-singular if

$$
1-\left[\begin{array}{c}
\mathbf{s}_{1} \\
\vdots \\
\mathbf{s}_{F}
\end{array}\right]^{\prime}\left[\begin{array}{ccc}
\psi_{1} \iota_{1} \mathbf{s}_{1}^{\prime}+I_{1} & \cdots & 0 \\
\vdots & \ddots & \vdots \\
0 & \cdots & \psi_{F} \iota_{F} \mathbf{s}_{F}^{\prime}+I_{F}
\end{array}\right]^{-1}\left[\begin{array}{c}
\rho_{1} \iota_{1} \\
\vdots \\
\rho_{F} \iota_{F}
\end{array}\right] \neq 0
$$

for any $\mathbf{p} \in \mathbb{R}^{J}$. Using the inverse (9), after some simple calculus this condition becomes

$$
1-\sum_{f=1}^{F} \frac{\rho_{f}}{1+\rho_{f}} \mathbf{s}_{f}^{\prime} \iota_{f} \neq 0 \text { for any } \mathbf{p} \in \mathbb{R}^{J}
$$

Since $\frac{\rho_{f}}{1+\rho_{f}}<1$

$$
\sum_{f=1}^{F} \frac{\rho_{f}}{1+\rho_{f}} \mathbf{s}_{f}^{\prime} \iota_{f}<\sum_{f=1}^{F} \mathbf{s}_{f}^{\prime} \iota_{f}=1-s_{0}<1
$$

This implies that

$$
1-\sum_{f=1}^{F} \frac{\rho_{f}}{1+\rho_{f}} \mathbf{s}_{f}^{\prime} \iota_{f}>0 \text { for any } \mathbf{p} \in \mathbb{R}^{J}
$$

and hence $\frac{\partial \mathbf{g}}{\partial \mathbf{p}^{\prime}}-I_{J}$ is non-singular, that is, $\operatorname{det}\left(\frac{\partial \mathbf{g}(\mathbf{p})}{\partial \mathbf{p}^{\prime}}-I_{J}\right) \neq 0$ for any $\mathbf{p} \in \mathbb{R}^{J}$.

\section{Appendix B}

Here we show that the pricing game implied by the standard logit case is neither supermodular nor log-supermodular. The definition of supermodularity from Milgrom and Roberts (1990) implies that the pricing game in the case of the standard logit is supermodular if the profit functions are twice continuously differentiable and for any $\mathbf{p}$

$$
\begin{aligned}
& \frac{\partial^{2} \Pi_{f}(\mathbf{p})}{\partial p_{j} \partial p_{h}} \geq 0 \text { for any } f \text { and } j, h \in G_{f}, j \neq h, \text { and } \\
& \frac{\partial^{2} \Pi_{f}(\mathbf{p})}{\partial p_{j} \partial p_{k}} \geq 0 \text { for any } f \text { and } j \in G_{f}, k \notin G_{f} .
\end{aligned}
$$

The pricing game is log-supermodular if the logarithms of the profit functions satisfy the above criteria. We show that the first inequality does not hold for the 
standard logit model. From Proposition 7 it follows that there is a unique price equilibrium, $\mathbf{p}^{*}$, and this solves the first-order conditions for profit maximization, that is, $\frac{\partial \Pi_{f}\left(\mathbf{p}^{*}\right)}{\partial p_{j}}=0$ for any $f$ and $j \in G_{f}$. Equation (5) implies that

$$
1-\alpha\left(p_{j}^{*}-c_{j}\right)+\alpha \Pi_{f}\left(\mathbf{p}^{*}\right)=0 .
$$

The second order derivative of the profit function for $j, h \in G_{f}, j \neq h$ is

$$
\frac{\partial^{2} \Pi_{f}(\mathbf{p})}{\partial p_{j} \partial p_{h}}=\frac{\partial s_{j}}{\partial p_{h}}\left(1-\alpha\left(p_{j}-c_{j}\right)+\alpha \Pi_{f}(\mathbf{p})\right)+\alpha s_{j} \frac{\partial \Pi_{f}(\mathbf{p})}{\partial p_{h}} .
$$

Computed at the equilibrium price, this is zero:

$$
\frac{\partial^{2} \Pi_{f}\left(\mathbf{p}^{*}\right)}{\partial p_{j} \partial p_{h}}=0
$$

due to $(10)$ and $\frac{\partial \Pi_{f}\left(\mathbf{p}^{*}\right)}{\partial p_{h}}=0$. We use this fact to show the following.

Proposition $\mathbf{9}$ For arbitrary $j, h \in G_{f}$ there exists a $\underline{\mathbf{p}}$ arbitrarily close to $\mathbf{p}^{*}$ such that
1. $\frac{\partial^{2} \Pi_{f}(\underline{\mathbf{p}})}{\partial p_{j} \partial p_{h}}<0 \quad$ and
2. $\frac{\partial^{2} \ln \Pi_{f}(\underline{\mathbf{p}})}{\partial p_{j} \partial p_{h}}<0$.

Proof. Take any $\varepsilon>0$ and define $\mathbf{p}$ such that

$$
\underline{p}_{j}=p_{j}^{*}+\varepsilon, \quad \underline{p}_{h}=p_{h}^{*}+\varepsilon \quad \text { and } \quad \underline{p}_{r}=p_{r}^{*} \quad \text { for all } r \neq j, h
$$

Due to Theorem $7 \mathbf{p}_{f}^{*}$ is a unique global maximum of $\Pi_{f}\left(\cdot, \mathbf{p}_{-f}^{*}\right)$. Thus for any $\varepsilon>0$ we have $\Pi_{f}(\underline{\mathbf{p}})<\Pi_{f}\left(\mathbf{p}^{*}\right)$. We observe another way of writing the second order derivatives:

$$
\frac{\partial^{2} \Pi_{f}(\mathbf{p})}{\partial p_{j} \partial p_{h}}=\alpha s_{j} s_{h}\left(2-\alpha\left(p_{j}-c_{j}\right)-\alpha\left(p_{h}-c_{h}\right)+2 \alpha \Pi_{f}(\mathbf{p})\right) .
$$

Then (11) implies that

$$
2-\alpha\left(p_{j}^{*}-c_{j}\right)-\alpha\left(p_{h}^{*}-c_{h}\right)+2 \alpha \Pi_{f}\left(\mathbf{p}^{*}\right)=0 .
$$


From the definition of $\underline{\mathbf{p}}$ we have

$2-\alpha\left(\underline{p}_{j}-c_{j}\right)-\alpha\left(\underline{p}_{h}-c_{h}\right)+2 \alpha \Pi_{f}(\underline{\mathbf{p}})<2-\alpha\left(p_{j}^{*}-c_{j}\right)-\alpha\left(p_{h}^{*}-c_{h}\right)+2 \alpha \Pi_{f}\left(\mathbf{p}^{*}\right)=0$.

Together with (12) this implies statement 1 of the proposition.

For showing statement 2 we write

$$
\frac{\partial^{2} \ln \Pi_{f}(\underline{\mathbf{p}})}{\partial p_{j} \partial p_{h}}=\frac{\frac{\partial^{2} \Pi_{f}(\underline{\mathbf{p}})}{\partial p_{j} \partial p_{h}} \Pi_{f}(\underline{\mathbf{p}})-\frac{\partial \Pi_{f}(\underline{\mathbf{p}})}{\partial p_{j}} \frac{\partial \Pi_{f}(\underline{\mathbf{p}})}{\partial p_{h}}}{\left(\Pi_{f}(\underline{\mathbf{p}})\right)^{2}}
$$

Because

$$
1-\alpha\left(\underline{p}_{j}-c_{j}\right)+\alpha \Pi_{f}(\underline{\mathbf{p}})<1-\alpha\left(p_{j}^{*}-c_{j}\right)+\alpha \Pi_{f}\left(\mathbf{p}^{*}\right)=0,
$$

it follows from (5) that $\frac{\partial \Pi_{f}(\underline{\mathbf{p}})}{\partial p_{j}}<0$, and similarly $\frac{\partial \Pi_{f}(\underline{\mathbf{p}})}{\partial p_{h}}<0$. Thus

$$
\frac{\partial \Pi_{f}(\underline{\mathbf{p}})}{\partial p_{j}} \frac{\partial \Pi_{f}(\underline{\mathbf{p}})}{\partial p_{h}}>0
$$

and therefore the right hand side of (13) is negative, which completes the proof.

We note that because $\underline{\mathbf{p}}$ is arbitrarily close to $\mathbf{p}^{*}$ we do not run the risk of having $\mathbf{p}$ outside the strategy sets of the firms, so the result is robust in this sense. Therefore we can safely claim that this pricing game is neither supermodular nor log-supermodular. 


\section{References}

Anderson, S.P. and A. de Palma, 1992, Multiproduct Firms: a Nested Logit Approach, Journal of Industrial Economics 40, 261-276.

Anderson, S.P., A. de Palma and J-F. Thisse, 1992, Discrete Choice Theory of Product Differentiation, MIT Press, Cambridge and London.

Berry, S., J. Levinsohn and A. Pakes, 1995, Automobile Prices in Market Equilibrium, Econometrica 63, 841-890.

Berry, S., O.B. Linton and A. Pakes, 2003, Limit Theorems for Estimating the Parameters of Differentiated Product Demand Systems, Working Paper.

Caplin, A. and B. Nalebuff, 1991, Aggregation and Imperfect Competition: On the Existence of Equilibrium, Econometrica 59, 25-59.

Dhrymes, P.J., 1984, Mathematics for Econometrics, Springer, New York.

Feenstra, R.C. and J.A. Levinsohn, 1995, Estimating Markups and Market Conduct with Multidimensional Product Attributes, Review of Economic Studies 62, $19-52$.

Gale, D. and H. Nikaido, 1965, The Jacobian Matrix and Global Univalence of Mappings, Mathematische Annalen 159, 81-93.

Kellogg, R.B., 1976, Uniqueness in the Schauder Fixed Point Theorem, Proceedings of the American Mathematical Society 60, 207-210.

Mas-Colell, A., 1985, The Theory of General Economic Equilibrium, Cambridge University Press, Cambridge.

Milgrom, P. and J. Roberts, 1990, Rationalizability, Learning, and Equilibrium in Games with Strategic Complementarities, Econometrica 58, 1255-1277. 
Mizuno, T., 2003, On the existence of a unique price equilibrium for models of product differentiation, International Journal of Industrial Organization 21, 76193.

Nevo, A., 2001, Measuring Market Power in the Ready-to-Eat Cereal Industry, Econometrica 69, 307-42.

Peitz, M., 2000, Aggregation in a Model of Price Competition, Journal of Economic Theory $\mathbf{9 0 , 1 - 3 8 . ~}$

Sándor, Z., 2001, Computation, Efficiency and Endogeneity in Discrete Choice Models, PhD thesis, University of Groningen.

Vives, X., 1999, Oligopoly Pricing, MIT Press, Cambridge. 